\title{
CNS relapse in patients with $D L B C L$ treated with lenalidomide plus R-CHOP (R2CHOP): analysis from two phase 2 studies
}

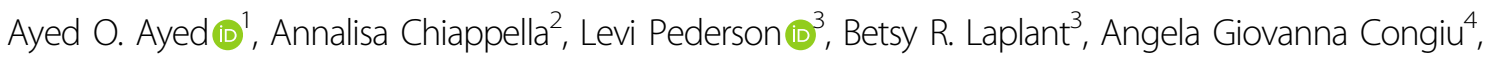 \\ Gianluca Gaidano ${ }^{5}$, Michele Spina ${ }^{6}$, Alessandro Re ${ }^{7}$, Federica Cavallo ${ }^{8}$, Gerardo Musuraca ${ }^{9}$, William R. Macon ${ }^{10}$, \\ Thomas Witzig ${ }^{1}$, Umberto Vitolo ${ }^{2}$ and Grzegorz S. Nowakowski ${ }^{1}$
}

\begin{abstract}
Central nervous system (CNS) relapse of diffuse large B-cell lymphoma (DLBCL) is a devastating event occurring in $\sim 5 \%$ of patients treated with R-CHOP. We hypothesized that adding lenalidomide to R-CHOP (R2CHOP) may decrease the risk of CNS relapse. We analyzed records for patients with DLBCL from two R2CHOP trials. We assessed variables pertinent to the CNS-International Prognostic Index (CNS-IPI) scoring system and classified patients into groups of low, intermediate, and high risk of CNS relapse. The 2-year CNS relapse rate for each risk group was estimated using the Kaplan-Meier method and compared with reported rates in cohorts treated with contemporary chemoimmunotherapy. A total of 136 patients were included. Mean age was 65 and median follow-up was 48.2 months. 10.3, 71.3, and 18.4\% of patients were classified into low, intermediate, and high-risk CNS-IPI groups, respectively. Only one of 136 patients developed CNS relapse, corresponding to an incidence of $0.7 \%$ and an estimated 2-year CNS relapse rate of $0.9 \%$ for the entire R2CHOP cohort. The estimated 2-year CNS relapse rates for the low, intermediate, and high-risk groups were 0, 0, and 5.0\%, respectively. Frontline therapy with R2CHOP in patients with $\mathrm{DLBCL}$ is associated with a lower-than-expected rate of CNS relapse.
\end{abstract}

\section{Introduction}

Relapse of aggressive non-Hodgkin lymphoma (NHL) in the central nervous system (CNS) is an uncommon but serious and potentially fatal event. Incidence rates of up to $10 \%$ have been reported ${ }^{1-4}$. The addition of rituximab to standard CHOP therapy (R-CHOP; cyclophosphamide, doxorubicin, vincristine, prednisone) has contributed to a reduction in both systemic and CNS relapse ${ }^{5-7}$. It is important to note however that the blood-brain barrier (BBB) renders the CNS a sanctuary site where penetration by standard chemoimmunotherapy is insufficient. Given

Correspondence: Grzegorz S. Nowakowski (Nowakowski.Grzegorz@mayo.edu) ${ }^{1}$ Division of Hematology, Mayo Clinic, Rochester, MN, USA

2Division of Hematology, Città della Salute e della Scienza Hospital and University, Torino, Italy

Full list of author information is available at the end of the article

on behalf of Fondazione Italiana Linfomi: Annalisa Chiappella, Angela Giovanna Congiu, Gianluca Gaidano, Michele Spina, Alessandro Re, Federica Cavallo, Gerardo Musuraca and Umberto Vitolo. the poor outcome of CNS relapse, there is clearly a need to incorporate agents that cross the $\mathrm{BBB}$ in upfront regimens to mitigate the risk of this event.

A number of factors are associated with an increased risk of developing CNS relapse in NHL such as older age, advanced stage, extranodal disease, elevated lactate dehydrogenase (LDH), and renal/adrenal involvement ${ }^{3,8}$. The CNS-International Prognostic Index (CNS-IPI) is a validated scoring system ${ }^{3}$ that risk-stratifies patients with diffuse large B-cell lymphoma (DLBCL) and may potentially help identify those at higher risk of developing CNS relapse.

Lenalidomide, an immunomodulatory agent that penetrates the CNS, has shown promise in relapsed/refractory $(\mathrm{R} / \mathrm{R})$ aggressive NHL, with well-demonstrated singleagent activity and tolerability ${ }^{9,10}$. Specifically, lenalidomide 
is active and well-tolerated in heavily pre-treated CNS lymphoma ${ }^{11}$. Combination therapy with lenalidomide (with bendamustine/rituximab (BR) or R-CHOP) has also been shown to be effective and tolerable in both the upfront and R/R settings ${ }^{12-14}$. Given its ability to cross the $\mathrm{BBB}$, we hypothesized that lenalidomide would lower the risk of CNS relapse when included in induction therapy. In this study, we evaluated and characterized the incidence of isolated CNS relapse in a combined cohort of DLBCL patients treated with upfront lenalidomide plus $\mathrm{R}-\mathrm{CHOP}$ (R2CHOP).

\section{Methods}

Patients with histologically confirmed DLBCL treated with $\mathrm{R} 2 \mathrm{CHOP}$ who were enrolled in two phase 2 trialsMayo Clinic (MC) $)^{15}$ (NCT00670358) and Fondazione Italiana Linfomi (FIL) ${ }^{12}$ (NCT00907348)-were included in this study. Several patients $(n=23)$ in the MC cohort whose histology excluded them from the final trial analysis (owing to histologic transformation or composite lymphoma) were included in this analysis, as they were allowed to remain on study following a protocol amendment. All patients had positron emission tomography (PET) imaging prior to and after completion of therapy, but not brain magnetic resonance imaging. Accordingly, patients were deemed to have no CNS involvement at baseline by clinical assessment and PET imaging only.

Data collected from patient records included age, gender, disease stage, status at last follow-up, cell of origin (COO) based on immunohistochemistry according to Hans' algorithm, LDH level, extranodal site involvement, Eastern Cooperative Oncology Group (ECOG) performance status, and administration of CNS prophylaxis. The protocol for each trial allowed intrathecal (IT) chemotherapy for prophylactic purposes per local practice (MC uses methotrexate $12 \mathrm{mg}$ IT for 6 doses, FIL uses methotrexate $12 \mathrm{mg}$, or $15 \mathrm{mg}$ IT for $4-6$ doses). No systemic CNS prophylaxis was allowed.

\section{Risk stratification}

The combined cohort of patients was stratified into three risk groups based on calculated CNS-IPI score. This scoring tool uses independent variables (age, stage, LDH level, ECOG performance status, extranodal sites, adrenal/kidney involvement) to classify patients into low (score 0-1), intermediate (score 2-3), and high (score 4-6) risk of CNS relapse ${ }^{3}$.

\section{Statistical methods}

Kruskal-Wallis or chi-square test was used to compare the three risk groups in terms of various clinical variables including age, stage, COO, LDH level, extranodal site involvement, ECOG performance status, and administration of CNS prophylaxis. The Kaplan-Meier method was used for time-to-event analyses. SAS ${ }^{\circledR}$ software (version 9.4 M3) was used for all statistical analyses.

\section{Comparison with other cohorts}

We compared the Kaplan-Meier estimated 2-year CNS relapse rates of $\mathrm{R} 2 \mathrm{CHOP}$ patients for each of the CNS-IPI risk groups (low, intermediate, and high), and also for the entire cohort, to rates published for patients treated with contemporary R-CHOP. The comparison cohorts included the R-CHOP-treated cohort of the Molecular Epidemiology Resource (MER) of the University of Iowa/ Mayo Clinic Specialized Program of Research Excellence database, as reported by Thanarajsingam and colleagues ${ }^{16}$, as well as two large DLBCL cohorts treated with contemporary chemoimmunotherapy, (German High-Grade Non-Hodgkin Lymphoma Study Group/MabThera International Trial (DSHNHL/MInT) and British Columbia Cancer Agency (BCCA) DLBCL cohorts), as reported by Schmitz et al. ${ }^{3}$ Data for the R-CHOP-treated cohort of the MER was obtained through direct correspondence and discussion with the authors of that study, whereas data pertaining to the CNS relapse rates for the DSHNHL/MInT and BCCA DLBCL cohorts was obtained directly from the publication for that report.

\section{Results}

A total of 136 patients with DLBCL (87 MC patients, 49 FIL patients) were included in this study (Table 1). Mean age was 65 and median follow-up in 104 patients that were still alive at the time of the analysis was 48.2 months (range: $2.1-88.5$ ).

When categorized into risk groups per the CNS-IPI score, 14 (10.3\%), 97 (71.3\%), and 25 (18.4\%) patients were classified into low, intermediate, and high-risk groups, respectively (Fig. 1). Comparison between these groups across different variables is shown in Table 2.

Of the 136 patients, only one developed isolated CNS relapse (which was parenchymal in nature), yielding an incidence rate of $0.007(0.7 \%)$. His disease at diagnosis was consistent with a GCB phenotype, extensive extranodal disease primarily involving multiple bony sites, and a calculated CNS-IPI score of 4 (high risk). Time to development of CNS relapse from diagnosis in this particular patient was 10 months and the relapse occurred 6 months after achieving a complete response (CR) by PET imaging. He was taken off protocol and salvaged with a high-dose methotrexate regimen followed by autologous stem cell transplantation.

The estimated 2-year CNS relapse rates by Kaplan-Meier method are $0.9 \%$ for the entire R2CHOP cohort and 0, 0, and 5.0\% for the low, intermediate, and high-risk groups, respectively. When compared with contemporary DLBCL patients treated with $\mathrm{R}-\mathrm{CHOP}$ from the MER database, the overall 2-year CNS relapse 
Table 1 Baseline patient characteristics

\begin{tabular}{|c|c|c|c|}
\hline & $\begin{array}{l}\text { FIL } \\
(n=49)\end{array}$ & $\begin{array}{l}\text { MC } \\
(n=87)\end{array}$ & $\begin{array}{l}\text { Total } \\
(n=136)\end{array}$ \\
\hline \multicolumn{4}{|l|}{ Age } \\
\hline Median & 69 & 65 & 68 \\
\hline Range & $(61.0-79.0)$ & $(19.0-87.0)$ & $(19.0-87.0)$ \\
\hline \multicolumn{4}{|l|}{ Clinical stage } \\
\hline 2 & $6(12.2 \%)$ & $13(14.9 \%)$ & $19(14.0 \%)$ \\
\hline 3 & $8(16.3 \%)$ & $21(24.1 \%)$ & $29(21.3 \%)$ \\
\hline 4 & 35 (71.4\%) & $53(60.9 \%)$ & $88(64.7 \%)$ \\
\hline \multicolumn{4}{|l|}{ Cell of origin } \\
\hline GCB & $16(32.7 \%)$ & $43(49.4 \%)$ & $59(43.4 \%)$ \\
\hline Non-GCB & $16(32.7 \%)$ & $34(39.1 \%)$ & $50(36.8 \%)$ \\
\hline NA & $17(34.7 \%)$ & $10(11.5 \%)$ & $27(19.9 \%)$ \\
\hline \multicolumn{4}{|c|}{ LDH above ULN } \\
\hline No & $26(53.1 \%)$ & $34(39.1 \%)$ & $60(44.1 \%)$ \\
\hline Yes & $23(46.9 \%)$ & $53(60.9 \%)$ & $76(55.9 \%)$ \\
\hline \multicolumn{4}{|c|}{ Extranodal sites } \\
\hline 0 or 1 & 31 (63.3\%) & $64(73.6 \%)$ & $95(69.9 \%)$ \\
\hline$>1$ site & $18(36.7 \%)$ & $23(26.4 \%)$ & $41(30.1 \%)$ \\
\hline \multicolumn{4}{|c|}{ Performance status } \\
\hline 0 & 17 (34.7\%) & $43(49.4 \%)$ & $60(44.1 \%)$ \\
\hline 1 & 25 (51.0\%) & $35(40.2 \%)$ & $60(44.1 \%)$ \\
\hline 2 & 7 (14.3\%) & $9(10.3 \%)$ & $16(11.8 \%)$ \\
\hline \multicolumn{4}{|c|}{ CNS prophylaxis } \\
\hline No & $30(61.2 \%)$ & 86 (98.9\%) & $116(85.3 \%)$ \\
\hline Yes & 19 (38.8\%) & $1(1.1 \%)$ & $20(14.7 \%)$ \\
\hline \multicolumn{4}{|l|}{ CNS-IPI score } \\
\hline Low & $0(0.0 \%)$ & $14(16.1 \%)$ & $14(10.3 \%)$ \\
\hline Intermediate & 37 (75.5\%) & 60 (69.0\%) & 97 (71.3\%) \\
\hline High & $12(24.5 \%)$ & $13(14.9 \%)$ & $25(18.4 \%)$ \\
\hline
\end{tabular}

GCB germinal center B-cell, NA not available, ULN upper limit of normal, MC Mayo Clinic, FIL Fondazione Italiana Linfomi

rate in the R2CHOP cohort is lower (0.9 vs. $1.8 \%$, Table 3). Similarly, the 2-year CNS relapse rates across risk groups, and overall, are lower for the R2CHOP cohort compared with the DLBCL cohorts from DSHNHL/MInT and BCCA (Table 3), as reported by Schmitz et al. ${ }^{3}$.

\section{Discussion}

CNS relapse in DLBCL continues to occur in a significant minority of patients and carries a poor prognosis even in the era of modern chemoimmunotherapy. Improvements in upfront regimens are needed to prevent

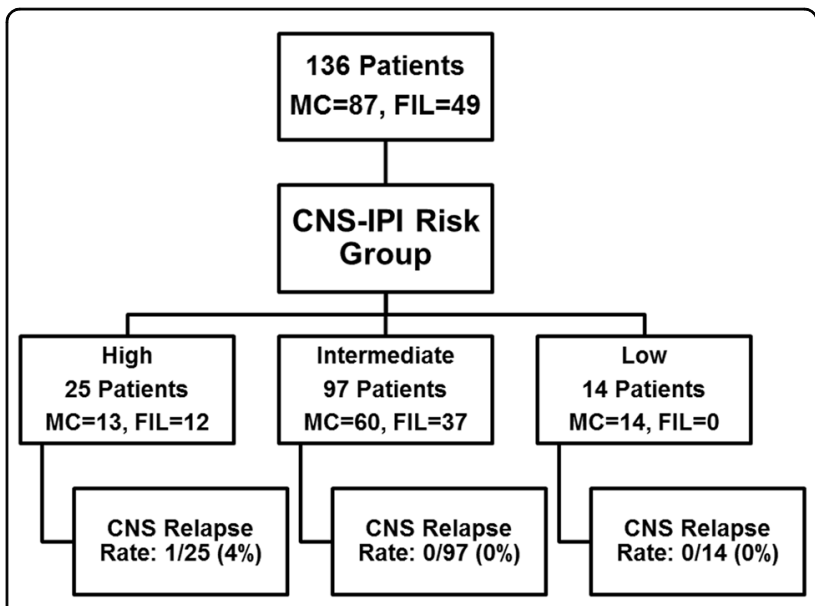

Fig. 1 Patient flow diagram. $M C=$ Mayo Clinic. $F I L=$ Fondazione Italiana Linfomi

lymphoma relapse in this sanctuary site. We report a critical observation in this analysis of a combined cohort of DLBCL patients treated with R2CHOP. Although CNS relapse rates with contemporary therapy have been roughly reported to range up to $5 \%{ }^{17}$, we note a considerably lower rate of $0.7 \%$. The median follow-up duration of 48.2 months is adequate to identify CNS events, as most cases of CNS relapse tend to occur within a few months from diagnosis ${ }^{4}$. It is worth noting that this lower-than-expected CNS relapse rate is observed in a population that is mostly intermediate and high risk $(n=$ $122,89.7 \%$ ) by CNS-IPI score.

Our analysis reveals considerable practice variation with regard to the use of prophylactic IT methotrexate, with significantly more patients having received IT chemotherapy in the FIL cohort compared with the MC cohort. This reflects regional and institutional differences in the administration of CNS prophylaxis, which is often based on the provider's judgement. This variation in practice is one of our study's limitations since it can contribute to confounding bias. Interestingly, however, several studies have suggested that IT chemoprophylaxis is ineffective in reducing the risk of CNS relapse ${ }^{1,18,19}$. As such, we believe that the lack of standardization in prophylactic IT methotrexate practice did not have a significant impact on our analysis. Another limitation of our study is the relatively small number of patients in the combined cohorts. Furthermore, neither trial was powered to assess for CNS events as CNS relapse was not a particular outcome measure in the study protocols.

There continues to be significant variability and controversy with regard to optimal CNS prophylaxis strategies. This lack of consensus is driven primarily by lack of strong evidence for benefit of current strategies and the difficulty of conducting definitive randomized studies in this area ${ }^{20}$. Recent practice trends tend to favor systemic 
Table 2 Baseline patient characteristics by CNS-IPI score

\begin{tabular}{|c|c|c|c|c|}
\hline & $\begin{array}{l}\text { High risk } \\
(n=25)\end{array}$ & $\begin{array}{l}\text { Intermediate risk } \\
(n=97)\end{array}$ & $\begin{array}{l}\text { Low risk } \\
(n=14)\end{array}$ & $P$ value \\
\hline \multicolumn{4}{|l|}{ Age } & 0.0014 \\
\hline Median & 69 & 69 & 56.5 & \\
\hline Range & $(55.0-83.0)$ & $(19.0-87.0)$ & $(24.0-79.0)$ & \\
\hline \multicolumn{4}{|c|}{ Clinical stage } & $<0.0001$ \\
\hline 2 & $0(0.0 \%)$ & $13(13.4 \%)$ & $6(42.9 \%)$ & \\
\hline 3 & $0(0.0 \%)$ & $25(25.8 \%)$ & $4(28.6 \%)$ & \\
\hline 4 & 25 (100.0\%) & $59(60.8 \%)$ & $4(28.6 \%)$ & \\
\hline \multicolumn{4}{|c|}{ Cell of origin } & 0.2353 \\
\hline GCB & $7(28.0 \%)$ & $47(48.5 \%)$ & $5(35.7 \%)$ & \\
\hline Non-GCB & $10(40.0 \%)$ & $35(36.1 \%)$ & $5(35.7 \%)$ & \\
\hline NA & $8(32.0 \%)$ & $15(15.5 \%)$ & $4(28.6 \%)$ & \\
\hline \multicolumn{4}{|c|}{ LDH above ULN } & $<0.0001$ \\
\hline No & $2(8.0 \%)$ & $45(46.4 \%)$ & $13(92.9 \%)$ & \\
\hline Yes & $23(92.0 \%)$ & $52(53.6 \%)$ & $1(7.1 \%)$ & \\
\hline \multicolumn{4}{|c|}{ Extranodal sites } & $<0.0001$ \\
\hline 0 or 1 & $2(8.0 \%)$ & 79 (81.4\%) & $14(100.0 \%)$ & \\
\hline$>1$ site & $23(92.0 \%)$ & $18(18.6 \%)$ & $0(0.0 \%)$ & \\
\hline \multicolumn{4}{|c|}{ Performance status } & $<0.0001$ \\
\hline 0 & $5(20.0 \%)$ & $45(46.4 \%)$ & $10(71.4 \%)$ & \\
\hline 1 & $9(36.0 \%)$ & $47(48.5 \%)$ & $4(28.6 \%)$ & \\
\hline 2 & $11(44.0 \%)$ & $5(5.2 \%)$ & $0(0.0 \%)$ & \\
\hline \multicolumn{4}{|c|}{ CNS prophylaxis } & $<0.0001$ \\
\hline No & $14(56.0 \%)$ & $88(90.7 \%)$ & $14(100.0 \%)$ & \\
\hline Yes & $11(44.0 \%)$ & $9(9.3 \%)$ & $0(0.0 \%)$ & \\
\hline
\end{tabular}

Based on CNS-IPI score, patients are classified into low (0-1), intermediate (2-3), or high (4-6) risk of CNS relapse

GCB germinal center B-cell, NA not available, ULN upper limit of normal intravenous (IV) methotrexate over IT therapy ${ }^{21,22}$. Although prophylactic IV methotrexate is gaining popularity, it tends to be costly and inconvenient and has the potential for serious toxicity. Our study suggests the potential prophylactic benefit of utilizing lenalidomide, an orally bioavailable CNS-penetrating agent, when combined with R-CHOP in the upfront setting. The two phase 2 trials analyzed in this study have already demonstrated safety and manageable toxicity of such a combination.

Further assessment of the utility of lenalidomide in reducing CNS relapse risk in DLBCL is warranted. Effective stratification of patients and identification of those at higher risk will likely involve both clinical and molecular parameters. At present, two phase 3 trials evaluating the combination of lenalidomide and R-CHOP in DLBCL patients are underway (ECOG1412 (NCT01856192) and ROBUST (NCT02285062)). Characterization of CNS events in these trials will be helpful in confirming the findings we noted in this analysis.

Our finding that frontline R2CHOP may lower the risk of CNS relapse in patients with DLBCL supports an ongoing trend in clinical studies where other novel small molecules are being investigated in the upfront setting in combination with R-CHOP, such as ibrutinib (NCT01855750), venetoclax (NCT02055820), and everolimus $^{23}$ (NCT01334502). The impact of such R(X)CHOP combinations $^{24}$ (where $\mathrm{X}$ represents a novel agent molecule) on CNS relapse is yet to be defined.

\section{Funding}

Celgene provided funding for the two phase 2 studies from which the data were obtained. Celgene had no role in study design, data collection, data analysis, data interpretation, or manuscript preparation.

\section{Author contributions}

A.O.A., A.C., T.W., U.V., and G.N.: designed research, analyzed and interpreted data, wrote manuscript. L.P., B.R.L.: performed statistical analysis, contributed to manuscript. A.G.C., G.G., M.S., A.R., F.C., G.M., and W.R.M.: contributed to data collection and research, contributed to manuscript.

Table 3 Estimated 2-year CNS relapse rate per risk group across cohorts

Two-year CNS relapse rates, estimated by Kaplan-Meier method

\begin{tabular}{|c|c|c|c|c|}
\hline Risk group & DSHNHL/MInT DLBCL cohort ${ }^{a}$ & BCCA DLBCL cohort $^{a}$ & MER R-CHOP cohort $^{b}$ & MC/FIL R2CHOP cohort \\
\hline Low & $0.8 \%$ (95\% Cl: 0.2-1.4\%) & $0.8 \%$ (95\% Cl: $0.0-1.6 \%)$ & $1.4 \%$ (95\% Cl: $0.5-3.7 \%)$ & 0\% (95\% Cl: 0.0-0.0\%) \\
\hline Intermediate & 2.9\% (95\% Cl: $1.5-4.3 \%)$ & 3.9\% (95\% Cl: $2.3-5.5 \%)$ & $2.2 \%(95 \% \mathrm{Cl}: 1.2-4.2 \%)$ & 0\% (95\% Cl: 0.0-0.0\%) \\
\hline High & $10.0 \%$ (95\% Cl: 5.7-14.3\%) & 12.0\% (95\% Cl: 7.9-16.1\%) & $1.1 \%$ (95\% Cl: 0.2-8.1\%) & $5.0 \%$ (95\% Cl: 0.0-14.1\%) \\
\hline Overall & Not available & $4.8 \%$ (95\% Cl: 3.6-6.0\%) & $1.8 \%$ (95\% Cl: $1.1-3.0 \%)$ & $0.9 \%$ (95\% Cl: $0.0-2.6 \%)$ \\
\hline
\end{tabular}

MC/FIL Mayo Clinic/Fondazione Italiana Linfomi, DSHNHL/MInT German High-Grade Non-Hodgkin Lymphoma Study Group/ MabThera International Trial, BCCA British Columbia Cancer Agency, MER Molecular Epidemiology Resource of the University of lowa/Mayo Clinic Specialized Program of Research Excellence.

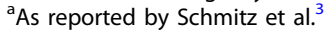

${ }^{b}$ As reported by Thanarajasingam et al. ${ }^{16}$ 


\section{Author details}

'Division of Hematology, Mayo Clinic, Rochester, MN, USA. ${ }^{2}$ Division of Hematology, Città della Salute e della Scienza Hospital and University, Torino, Italy. ${ }^{3}$ Department of Health Sciences Research, Mayo Clinic, Rochester, MN, USA. ${ }^{4}$ Department of Hematology, IRCCS San Martino Hospital and University, Genova, Italy. ${ }^{5}$ Division of Hematology, Department of Translational Medicine, Amedeo Avogadro University of Eastern Piedmont, Novara, Italy. ${ }^{6}$ Division of Medical Oncology A, National Cancer Institute, Aviano, Italy. ${ }^{7}$ Department of Hematology, ASST Spedali Civili di Brescia, Brescia, Italy. ${ }^{8}$ Department of Hematology, University of Torino, Torino, Italy. ${ }^{9}$ Department of Hematology, Istituto Scientifico Romagnolo Per Lo Studio E La Cura Dei Tumori, Meldola (FC), Meldola, Italy. ${ }^{10}$ Department of Laboratory Medicine and Pathology, Mayo Clinic, Rochester, MN, USA

\section{Institutional review board committee approval}

The two phase 2 trials from which the data were collected have been approved by the local institutional review board or ethics committee of each participating site. All research involving human subjects was conducted in accordance with the Declaration of Helsinki. Informed consent was obtained from all patients participating in the clinical trials.

\section{Conflict of interest}

AC: advisory board: Celgene; honoraria for lectures: Amgen, Celgene, Janssen, Nanostring, Pfizer, Roche, Teva. GG: consultancy: Roche, Karyopharm, Morphosys, Gilead, Janssen, Novartis; honoraria: Roche, Karyopharm, Morphosys, Gilead, Janssen, Novartis; speakers bureau: Roche, Gilead, Janssen, Novartis. MS: advisory board: Teva, Mundipharma; speaker fees: Teva, Mundipharma. FC: honoraria: Celgene, Onyx, Janssen. UV: advisory board: Roche, Celgene, Janssen; honoraria for lectures: Roche, Celgene, Takeda, Gilead, Janssen, Mundipharma; research funding: Roche, Celgene. GN: consultancy: Bayer; research funding: Bayer, Celgene, Morphosys. A.O.A., L.P., B.R.L., A.G.C., A.R., G.M., W.R.M., and T.W.: declare no conflict of interest.

\section{Publisher's note}

Springer Nature remains neutral with regard to jurisdictional claims in published maps and institutional affiliations.

Received: 28 August 2017 Revised: 18 May 2018 Accepted: 25 May 2018 Published online: 26 June 2018

\section{References}

1. Boehme, V., Schmitz, N., Zeynalova, S., Loeffler, M. \& Pfreundschuh, M. CNS events in elderly patients with aggressive lymphoma treated with modern chemotherapy (CHOP-14) with or without rituximab: an analysis of patients treated in the RICOVER-60 trial of the German High-Grade Non-Hodgkin Lymphoma Study Group (DSHNHL). Blood 113, 3896-3902 (2009).

2. Schmitz, N. et al. CNS disease in younger patients with aggressive B-cell lymphoma: an analysis of patients treated on the Mabthera International Trial and trials of the German High-Grade Non-Hodgkin Lymphoma Study Group. Ann. Oncol. 23, 1267-1273 (2012).

3. Schmitz, N. et al. CNS international prognostic index: a risk model for CNS relapse in patients with diffuse large B-cell lymphoma treated with R-CHOP. J. Clin. Oncol. 34, 3150-3156 (2016).

4. Bernstein, S. H. et al. Natural history of CNS relapse in patients with aggressive non-Hodgkin's lymphoma: a 20-year follow-up analysis of SWOG 8516-The Southwest Oncology Group. J. Clin. Oncol. 27, 114-119 (2009).

5. Villa, D. et al. Incidence and risk factors for central nervous system relapse in patients with diffuse large B-cell lymphoma: the impact of the addition of rituximab to CHOP chemotherapy. Ann. Oncol. 21, 1046-1052 (2010).
6. Feugier, P. et al. Incidence and risk factors for central nervous system occurrence in elderly patients with diffuse large-B-cell lymphoma: influence of rituximab. Ann. Oncol. 15, 129-133 (2004).

7. Abramson, J. S. High-dose chemotherapy and autologous stem cell transplantation for secondary central nervous system lymphoma: many are called, but few are chosen. Haematologica 98, 662-664 (2013).

8. Boehme, $\mathrm{V}$. et al. Incidence and risk factors of central nervous system recurrence in aggressive lymphoma--a survey of 1693 patients treated in protocols of the German High-Grade Non-Hodgkin's Lymphoma Study Group (DSHNHL). Ann. Oncol. 18, 149-157 (2007).

9. Witzig, T. E. et al. An international phase II trial of single-agent lenalidomide for relapsed or refractory aggressive B-cell non-Hodgkin's lymphoma. Ann. Oncol. 22, 1622-1627 (2011).

10. Wiernik, P. H. et al. Lenalidomide monotherapy in relapsed or refractory aggressive non-Hodgkin's lymphoma. J. Clin. Oncol. 26, 4952-4957 (2008).

11. Houillier, C. et al. Lenalidomide monotherapy as salvage treatment for recurrent primary CNS lymphoma. Neurology 84, 325-326 (2015).

12. Vitolo, U. et al. Lenalidomide plus R-CHOP21 in elderly patients with untreated diffuse large B-cell lymphoma: results of the REAL07 open-label, multicentre, phase 2 trial. Lancet Oncol. 15, 730-737 (2014).

13. Hitz, F. et al. Rituximab, bendamustine and lenalidomide in patients with aggressive B-cell lymphoma not eligible for anthracycline-based therapy or intensive salvage chemotherapy - SAKK 38/08. Br. J. Haematol. 174, 255-263 (2016).

14. Wang, M. et al. Oral lenalidomide with rituximab in relapsed or refractory diffuse large cell, follicular and transformed lymphoma: a phase II clinical trial. Leukemia 27, 1902-1909 (2013).

15. Nowakowski, G. S. et al. Lenalidomide combined with R-CHOP overcomes negative prognostic impact of non-germinal center B-cell phenotype in newly diagnosed diffuse large B-Cell lymphoma: a phase II study. J. Clin. Oncol. 33, 251-257 (2015).

16. Thanarajasingam G., et al. Event-free survival at 24 months captures central nervous system relapse of systemic diffuse large B-cell lymphoma in the immunochemotherapy era. Br. J. Haematol. (2017) [Epub ahead of print].

17. Zhang, J., Chen, B. \& Xu, X. Impact of rituximab on incidence of and risk factors for central nervous system relapse in patients with diffuse large B-cell lymphoma: a systematic review and meta-analysis. Leuk. Lymphoma 55, 509-514 (2014).

18. Murawski, N. et al. The role of radiotherapy and intrathecal CNS prophylaxis in extralymphatic craniofacial aggressive B-cell lymphomas. Blood 124, 720-728 (2014).

19. Chua, S. L. et al. Intrathecal chemotherapy alone is inadequate central nervous system prophylaxis in patients with intermediate-grade non-Hodgkin's lymphoma. Leuk. Lymphoma 43, 1783-1788 (2002).

20. Siegal, T. \& Goldschmidt, N. CNS prophylaxis in diffuse large B-cell lymphoma: if, when, how and for whom? Blood. Rev. 26, 97-106 (2012).

21. Schmitz, N. Nickelsen, M. \& Savage, K. J. Central nervous system prophylaxis for aggressive B-cell lymphoma: who, what, and when? Hematol. Oncol. Clin. North. Am. 30, 1277-1291 (2016).

22. Penalver, F. J. et al. Guidelines for diagnosis, prevention and management of central nervous system involvement in diffuse large B-cell lymphoma patients by the Spanish Lymphoma Group (GELTAMO). Haematologica 102, 235-245 (2017).

23. Johnston, P. B. et al. Everolimus combined with R-CHOP-21 for new, untreated, diffuse large B-cell lymphoma (NCCTG 1085 [Alliance]): safety and efficacy results of a phase 1 and feasibility trial. Lancet Haematol. 3, e309-e316 (2016).

24. Vaidya, R. \& Witzig, T. E. Prognostic factors for diffuse large B-cell lymphoma in the R(X)CHOP era. Ann. Oncol. 25, 2124-2133 (2014). 